\title{
Correction to: Extracorporeal membrane oxygenation for COVID-19: a systematic review and meta-analysis
}

\author{
Kollengode Ramanathan ${ }^{1,2^{*}+}$ (D), Kiran Shekar ${ }^{3,4,5,6 \dagger}$, Ryan Ruiyang Ling ${ }^{1}$, Ryan P. Barbaro ${ }^{7,8}$, Suei Nee Wong ${ }^{1}$, \\ Chuen Seng Tan ${ }^{1,9}$, Bram Rochwerg ${ }^{10,11}$, Shannon M. Fernando ${ }^{12}$, Shinhiro Takeda ${ }^{13}$, Graeme MacLaren ${ }^{1,2}$, \\ Eddy Fan ${ }^{14}$ and Daniel Brodie ${ }^{15,16}$
}

\section{Correction to: Crit Care (2021) 25:211.}

https://doi.org/10.1186/s13054-021-03634-1

Following publication of the original article [1], the authors identified errors in Tables 1, 2, the Additional file 2: Figure S1 and Additional file 3: Figure S2. The correct Tables are is given hereafter and an explanation on the Additional file Figure S1 and Figure S2.

Table 1 currently reads: Takeda et al. had 237 number of patients.

Table 1 should read: Takeda et al. had 370 patients ; 81.4\% (301 patients) were males and 343 required VV ECMO.

Table 2 currently reads Takeda et al. reported mortality in 67 patients.

Table 2 should read as Takeda et al. reported mortality in 120 patients with mean duration of $18.4 \pm 15.2$ days on ECMO.
The authors want to note that the forrest plot calculations in Fig. 2 was based on 370 patients. The article was written based on the forrest plot results (Fig. 2), which is the correct version.

The supplementary Figures 1 and 2 represent subgroup analysis based on 237 patients. However, on reanalysis, the final analysis results mostly remained the same, except for the confidence intervals, we have explained this in great detail in our rebuttal letter to Hoechter et al. [2]. The mortality on VV ECMO was 35.7\% (95\% CI: 30.8-40.7) while the regional mortality in Asia was 43.3\% (95\% CI: 28.9-58.3\%).

All the changes that were requested are implemented in this correction and the original article [1] has been corrected. 
Table 1 Demographics of the included studies

\begin{tabular}{|c|c|c|c|c|c|c|}
\hline First Author & Country & $\begin{array}{l}\text { Number of } \\
\text { patients }\end{array}$ & Male patients $\mathrm{n}(\%)$ & Age* & VV ECMO & P/F ratio* \\
\hline Alnababteh & United States of America & 13 & $8(61.5)$ & $44.54 \pm 9.49$ & 13 & $97.96 \pm 49.87$ \\
\hline Akhtar & UK & 18 & $16(88.9)$ & $47.3 \pm 0.9$ & 18 & NR \\
\hline Barbaro & International & 1035 & $764(73.8)$ & $49(41-57)$ & 978 & $72(59-94)$ \\
\hline Charlton & UK & 34 & $27(79.4)$ & $46.3 \pm 7.5$ & 34 & $64.5(54.7-74.3)$ \\
\hline Cousin & France & 30 & $24(80)$ & $33.33 \pm 7.00$ & 30 & $69 \pm 9.34$ \\
\hline Falcoz & France & 17 & $16(94.1)$ & $56[30-76]$ & 16 & $71[52-134]$ \\
\hline Guihaire & France & 24 & $20(83.3)$ & $48.8 \pm 8.9$ & 24 & $67[52-78]$ \\
\hline Huette & France & 12 & $12(100)$ & $62(58-64)$ & 14 & $76(66-83)$ \\
\hline Jackel & Germany & 15 & $11(73.3)$ & $60.8(54.2-67)$ & 15 & $63.7(51.9-94.5)$ \\
\hline Jang & Korea & 19 & $15(79)$ & $63 \pm 4.81$ & 16 & $97.7 \pm 61.11$ \\
\hline Le Breton & France & 13 & $10(77)$ & $49.31 \pm 7.75$ & 13 & $60.62 \pm 15.23$ \\
\hline Jozwiak & France & 11 & $7(63.6)$ & $50(38-59)$ & 11 & $68(58-89)$ \\
\hline Masur & United States of America & 12 & $8(66.7)$ & $53.83 \pm 13.18$ & NR & NR \\
\hline Mustafa & United States of America & 40 & $30(75)$ & $48.4 \pm 1.5$ & 40 & $68.9 \pm 3.1$ \\
\hline Roedl & Germany & 20 & $N R$ & $N R$ & 20 & NR \\
\hline Schmidt & France & 83 & $61(73.5)$ & $48.0 \pm 11.0$ & 81 & $62 \pm 18$ \\
\hline Shih & USA & 37 & $27(73.0)$ & $51(40-59)$ & 37 & $95(73-147)$ \\
\hline Takeda & Japan & 370 & $301(81.4)$ & NR & 343 & NR \\
\hline Yang & China & 21 & $12(57.1)$ & $58.5(42.75-67.25)$ & 21 & $60(55.6-72)$ \\
\hline Zayat & Germany & 17 & $11(64.7)$ & $57(53-62)$ & 17 & NR \\
\hline Zeng & China & 12 & $11(91.7)$ & $50.9 \pm 13.5$ & NR & $N R$ \\
\hline Zhang & UK & 43 & $33(76.7)$ & $46(35.5-52.5)$ & 43 & $67.5(58.9-77.8)$ \\
\hline
\end{tabular}

VV-ECMO, venovenous extracorporeal membrane oxygenation; $\mathrm{P} / \mathrm{F}$, partial pressure of arterial oxygen to fraction of inspired oxygen ratio $[\mathrm{PaO} 2 / \mathrm{FiO} 2] ; \mathrm{NR}$, not reported

* Age and $\mathrm{P} / \mathrm{F}$ ratio reported as mean $\pm \mathrm{SD}$, median (interquartile range) or median [range]

Table 2 Outcomes of the included studies

\begin{tabular}{|c|c|c|c|c|c|c|}
\hline First author & Mortality & Survivors & Not discharged & Still on ECMO & Complications on ECMO & Days on ECMO* \\
\hline Alnababteh & 6 & 4 & 3 & NR & $\begin{array}{l}3 \text { mechanical } \\
7 \text { haemorrhagic } \\
6 \text { renal } \\
4 \text { pulmonary } \\
2 \text { infectious } \\
3 \text { metabolic } \\
2 \text { limb }\end{array}$ & $12.85 \pm 6.04$ \\
\hline Akhtar & 4 & 14 & 0 & 0 & & $17.7 \pm 9.4$ \\
\hline Barbaro & 380 & 588 & 67 & 31 & $\begin{array}{l}295 \text { mechanical } \\
69 \text { neurologic } \\
444 \text { renal }\end{array}$ & $13.9(7.8-23.3)$ \\
\hline Charlton & 16 & 18 & 0 & 0 & & \\
\hline Cousin & 16 & NR & NR & NR & $\begin{array}{l}8 \text { mechanical } \\
27 \text { haemorrhagic } \\
4 \text { neurologic } \\
15 \text { renal } \\
2 \text { pulmonary } \\
4 \text { infectious } \\
3 \text { limb }\end{array}$ & $10.67 \pm 5.45$ \\
\hline
\end{tabular}


Table 2 (continued)

\begin{tabular}{|c|c|c|c|c|c|c|}
\hline First author & Mortality & Survivors & Not discharged & Still on ECMO & Complications on ECMO & Days on ECMO* \\
\hline Falcoz & 6 & 10 & 1 & 0 & $\begin{array}{l}7 \text { mechanical } \\
62 \text { haemorrhagic } \\
1 \text { neurologic } \\
12 \text { renal } \\
1 \text { cardiovascular } \\
3 \text { pulmonary } \\
10 \text { infectious } \\
1 \text { others (thrombocytopenia) }\end{array}$ & $9[0-16]$ \\
\hline Guihaire & 4 & 16 & 4 & 3 & $\begin{array}{l}18 \text { mechanical } \\
1 \text { neurologic } \\
10 \text { pulmonary } \\
3 \text { infectious } \\
1 \text { other (mesenteric ischemia) }\end{array}$ & $19.0 \pm 10.1$ \\
\hline Huette & 4 & 8 & 0 & 0 & $\begin{array}{l}5 \text { mechanical } \\
3 \text { hemorrhagic } \\
8 \text { renal } \\
1 \text { cardiovascular } \\
4 \text { pulmonary } \\
10 \text { infectious } \\
3 \text { others ( } 2 \text { liver failure, } 1 \text { HIT) }\end{array}$ & $12(9-22)$ \\
\hline Jackel & 8 & 7 & 0 & 0 & & \\
\hline Jang & 10 & 4 & 5 & 2 & $\begin{array}{l}5 \text { neurologic } \\
9 \text { renal } \\
1 \text { cardiovascular } \\
6 \text { pulmomary }\end{array}$ & $17.27 \pm 16.42$ \\
\hline Jozwiak & 6 & 5 & 0 & 0 & & \\
\hline Le Breton & 2 & 11 & 0 & 0 & $\begin{array}{l}2 \text { mechanical } \\
3 \text { hemorrhagic } \\
2 \text { infectious }\end{array}$ & $14.53 \pm 8.84$ \\
\hline Masur & 5 & 1 & 6 & NR & 6 neurologic & 9.60 \\
\hline Mustafa & 6 & 29 & 5 & 2 & 10 pulmonary & $29.9 \pm 3.6$ \\
\hline Roedl & 13 & 7 & 0 & 0 & & \\
\hline Schmidt & 25 & 38 & 20 & 5 & $\begin{array}{l}25 \text { mechanical } \\
45 \text { hemorrhagic } \\
5 \text { neurologic } \\
38 \text { renal } \\
11 \text { cardiovascular } \\
50 \text { pulmonary } \\
129 \text { infectious } \\
7 \text { others ( } 5 \text { thrombocytopenia, } 2 \text { HIT) }\end{array}$ & $20(10-40)$ \\
\hline Shih & 16 & 21 & 0 & 0 & & \\
\hline Takeda & 120 & NR & NR & NR & NA & $18.4 \pm 15.2$ \\
\hline Yang & 12 & 6 & 3 & 0 & $\begin{array}{l}3 \text { hemorrhagic } \\
8 \text { renal } \\
8 \text { cardiovascular } \\
6 \text { pulmonary } \\
3 \text { infectious }\end{array}$ & NR \\
\hline Zayat & 8 & 9 & 0 & 0 & & \\
\hline Zeng & 5 & NR & 7 & 4 & NA & $11.3 \pm 7.8$ \\
\hline Zhang & 14 & 29 & 0 & 0 & & \\
\hline
\end{tabular}

ECMO, extracorporeal membrane oxygenation; NR, not reported

*Days on $\mathrm{ECMO}$ reported as mean $\pm \mathrm{SD}$, median (interquartile range) or median [range] 


\begin{abstract}
Author details
${ }^{1}$ Yong Loo Lin School of Medicine, National University of Singapore, Singapore, Singapore. ${ }^{2}$ Cardiothoracic Intensive Care Unit, National University Heart Centre, National University Hospital, Singapore 119228 , Singapore. ${ }^{3}$ Adult Intensive Care Services, Prince Charles Hospital, Brisbane, QLD, Australia. ${ }^{4}$ Queensland University of Technology, Brisbane, Australia. ${ }^{5}$ University of Queensland, Brisbane, Australia. ${ }^{6}$ Bond University, Gold Coast, QLD, Australia. ${ }^{7}$ Division of Paediatrics Critical Care Medicine, University of Michigan, Ann Arbor, USA. ${ }^{8}$ Child Health Evaluation and Research Center, University of Michigan, Ann Arbor, MI, USA. ${ }^{9}$ Saw Swee Hock School of Public Health, National University of Singapore, Singapore, Singapore. ${ }^{10}$ Department of Medicine, Division of Critical Care, McMaster University, Hamilton, ON, Canada. ${ }^{11}$ Department of Health Research Methods, Evidence and Impact, McMaster University, Hamilton, ON, Canada. ${ }^{12}$ Division of Critical Care, Department of Medicine, University of Ottawa, Ottawa, ON, Canada. ${ }^{13}$ Japan ECMOnet for COVID-19 \& President, Kawaguchi Cardiovascular and Respiratory Hospital, Saitama, Japan. ${ }^{14}$ Interdepartmental Division of Critical Care Medicine, University of Toronto, Toronto, Canada. ${ }^{15}$ Department of Medicine, Columbia University College of Physicians and Surgeons, New York, NY, USA. ${ }^{16}$ Center for Acute Respiratory Failure, New York-Presbyterian Hospital, New York, NY, USA.
\end{abstract}

Published online: 27 October 2021

\section{References}

1. Ramanathan K, Shekar K, Ling RR, et al. Extracorporeal membrane oxygenation for COVID-19: a systematic review and meta-analysis. Crit Care. 2021;25:211. https://doi.org/10.1186/s13054-021-03634-1.

2. Hoechter DJ, Becker-Pennrich AS, Geisler BP, et al. Letter to the editor regarding Extracorporeal membrane oxygenation for COVID-19: a systematic review and meta-analysis. Crit Care. 2021. https://doi.org/10 1186/s13054-021-03702-6.

\section{Publisher's Note}

Springer Nature remains neutral with regard to jurisdictional claims in published maps and institutional affiliations.
Ready to submit your research? Choose BMC and benefit from:

- fast, convenient online submission

- thorough peer review by experienced researchers in your field

- rapid publication on acceptance

- support for research data, including large and complex data types

- gold Open Access which fosters wider collaboration and increased citations

- maximum visibility for your research: over $100 \mathrm{M}$ website views per year

At BMC, research is always in progress.

Learn more biomedcentral.com/submissions 